\title{
Neurological presentation predicting immune thrombotic thrombocytopenic purpura outcome
}

\author{
Shaimaa El-Ashwah ${ }^{1,{ }^{*}}$, Esraa Jamal ${ }^{1}$, Rasha Samir Shemies ${ }^{2}$, Metwaly Ibrahim Mortada ${ }^{3}$, \\ Mayada A. Ghannam ${ }^{3}$, Yasmine Shaaban ${ }^{1}$ \\ ${ }^{1}$ Clinical Hematology Unit, Internal Medicine Department, Faculty of Medicine, Mansoura University, Mansoura, Egypt \\ ${ }^{2}$ Mansoura Nephrology and Dialysis Unit, Faculty of Medicine, Mansoura University, Mansoura, Egypt \\ ${ }^{3}$ Hematology Unit, Clinical Pathology Department, Faculty of Medicine, Mansoura University, Mansoura, Egypt
}

\section{Abstract}

Introduction: Immune thrombotic thrombocytopenic purpura (iTTP) is a rare disorder caused by acquired autoantibodies to a disintegrin and metalloprotease with thrombospondin-1 motifs (ADAMTS-13) that normally cleaves von Willebrand factor macromolecules. It is manifested by microangiopathic hemolytic anemia, systemic microvascular thrombi formation, and subsequent end-organ ischemia (renal and neurological manifestations). Early diagnosis and management resulted in improving the survival rate. This is a retrospective study conducted to describe the clinical characteristics of patients diagnosed with iTTP, their survival, and prognostic factors affecting it.

Methods: We included adult patients who met the diagnostic criteria of iTTP between 2016 and 2019. Based on PLASMIC score for TTP, our patients ranged from 6 to 7. ADAMTS-13 testing was not done because of financial issues.

Results: A total of 21 patients were included in this study. The median age of the studied patients was 30.45 years, and $81 \%$ of them were female. The most common clinical feature was fever (57.1\%), followed by bleeding manifestations (52.4\%), neurological manifestations (47.6\%), renal impairment (42.9\%), and cardiac manifestations (9.5\%). There were a total of 4 deaths (19.04\%). The overall survival was correlated significantly with neurological manifestations and PLASMIC scores ( $p=0.02,0.012$, respectively).

Conclusions: Our report reinforces that iTTP is not mandatory to be presented with classic pentad. Using PLASMIC score could help in the diagnosis and prediction of survival, and we strongly suggest that the absence of neurological manifestations results in better overall survival. Therapeutic plasma exchange should be started as soon as possible once iTTP is suspected. Rituximab has an important role in improving treatment outcomes.

Key words: ADAMTS13, rituximab, thrombotic microangiopathy, thrombotic thrombocytopenic purpura

Acta Haematologica Polonica 2021; 52, 1: 48-53

\section{Introduction}

Thrombotic thrombocytopenic purpura (TTP) is a life-threatening thrombotic microangiopathy associated with a severe deficiency in ADAMTS13 (a disintegrin and metalloprotease with thrombospondin-1 motifs; $13^{\text {th }}$ member of the family), the enzyme that cleaves the ultra-large von Willebrand factor (vWF) multimers in human's plasma [1].
ADAMTS-13 deficiency is caused by a mutational defect in the ADAMTS-13 gene (congenital TTP) or immune-mediated (iTTP) by circulating autoantibodies against ADAMTS-13 that may be associated with a prior immune "trigger" such as pregnancy, history of infection, systemic disorders, and drugs [2]. ITTP is more common in the $4^{\text {th }}$ decade, with female to male ratio of 3:2 [3]. TTP must be suspected and confirmed by laboratory testing following its clinical
*Address for correspondence: Shaimaa El-Ashwah, Lecturer

of Clinical Hematology, Faculty of Medicine, Mansoura University,

Mansoura 35516, Egypt,

e-mail: shaimaelashwah@gmail.com

Received: 02.07.2020

Accepted: 03.09.2020
PTHiT Copyright $\odot 2021$

The Polish Society of Haematologists and Transfusiologists,

Insitute of Haematology and Transfusion Medicine.

All rights reserved. 
presentation. The "classical" pentad that includes thrombocytopenia, MAHA, fever, renal failure, and neurological manifestations is uncommon. Gastrointestinal symptoms, such as vomiting, diarrhea, and abdominal pain are frequent; however, myocardial ischemia, pancreatitis, and less commonly, a pulmonary injury may also occur [3, 4].

Advanced age, cardio-vascular system affection, and high lactate dehydrogenase (LDH) values have been correlated with high mortality risk. Management of iTTP involves proceeding safely and quickly initiating the immunomodulating agents targeting the lymphocytes responsible for autoantibodies production alongside a treatment backbone of therapeutic plasma exchange (TPEx), which helps to replete ADAMTS-13 and remove vWF and autoantibodies [5]. Untreated iTTP is associated with mortality as high as 90\%; with TPEx, mortality can be reduced to $20 \%$ [6]. ISTH guidelines recommend using corticosteroids in addition to TPEx for treatment of first acute episode and relapses of iTTP. Meanwhile, adding rituximab will be effective in the treatment of known comorbid autoimmune disease [7].

Recently, caplacizumab (anti-vWF nanobody), blocks the adhesion of platelets to VWF and prevents the formation of microvascular thrombi [8]. It is recommended to be used early at the time of an acute TTP event. However, it neither corrects the level of plasma ADAMTS-13 nor does it suppress the autoantibodies to ADAMTS-13. Thus, other immunosuppressive therapies use steroids and rituximab that are still required to control the underlying disease process [7-9].

In this study, we demonstrate the basic clinic-pathological features of TTP patients emphasizing the pattern and outcome of the disease.

\section{Material}

\section{Patients and methodology}

All data from iTTP patients referred to Oncology Center and Mansoura University from 2016 to 2019 were analyzed. We diagnosed our cases based on negative coombs test hemolytic anemia and found the presence of schistocytes in peripheral blood film stained with Leishman stain, acute peripheral thrombocytopenia $\left(<150 \times 10^{3} / \mathrm{mm}^{3}\right)$, reticulocyte counting by brilliant cresyl blue staining plus or minus the rest of the characteristic pentad. A workup including history, physical examination, radiological studies, serum LDH, liver function, renal function test, viral marker, antinuclear antibody, anti-double-stranded DNA antibody to exclude secondary causes of iTTP as drugs, pregnancy, autoimmune diseases, malignancies, HIV, and other infections. ADAMTS-13 testing was not done because of financial issues.

We retrospectively applied the PLASMIC score to stratify the cases (Table I). The PLASMIC score is a seven-item score with one point is assigned for each item. The risk stratification categories are as follows: low-risk (0-4),
Table I. PLASMIC scoring system

\begin{tabular}{|l|c|}
\hline Variables & Points \\
\hline Platelet count $<30 \times 10^{9} / \mathrm{L}$ & 1 \\
\hline Hemolysis present: & 1 \\
\hline - reticulocyte count $>2.5 \%$, or & \\
\hline - haptoglobin undetectable, or & \\
\hline indirect bilirubin $>2.0 \mathrm{mg} / \mathrm{dL}$ & \\
\hline No active cancer & 1 \\
\hline No history of solid organ or stem cell transplant & 1 \\
\hline Mean corpuscular volume (MCV) <90 & 1 \\
\hline International normalized ratio (INR) <1.5 & 1 \\
\hline Creatinine <2.0 mg/dL & 1
\end{tabular}

intermediate-risk (5), and high-risk (6-7). Internal and external validation studies revealed that the frequency of severe ADAMTS-13 deficiency is $0-4 \%, 5-24 \%$, and $62-82 \%$ in all risk categories, respectively [10].

Treatment response was defined according to international recommendations as a complete response (CR), which was defined as the absence of any clinical manifestations and normalization of platelet count for $\geq 2$ days. No doubling of platelet count after four days of standard intensive therapy and persistently high LDH levels was considered as a refractory disease. Relapse was the reappearance of clinical features of iTTP for at least 2 days [11].

\section{Statistical analysis}

Data were analyzed on a personal computer running SPSS21 for Windows (Statistical Package for Social Scientists) Release 18. A 2-tailed $p$-value of $<0.05$ was statistically significant. For descriptive statistics of qualitative variables, the frequency distribution procedure was run with the calculation of the number of cases and percentages. For descriptive statistics of quantitative variables, mean, standard deviation (SD), and range were used to describe the central tendency and dispersion as appropriate. The overall survival was estimated through the Kaplan-Meier test.

\section{Results}

This study was conducted on 21 patients. The mean age of the studied patients was 30.45 years, and $81 \%$ of them were female. The most common clinical feature was fever (57.1\%), followed by bleeding manifestations (52.4\%), neurological manifestations (47.6\%) in form of headache, slurred speech, seizures, confusion up to coma, renal impairment (42.9\%), and cardiac manifestations (9.5\%) in form of arrhythmia and myocardial infarction. The mean values at presentation were consistent with microangiopathic hemolytic anemia (Table II). 
Table II. Basic descriptive date of studied group

\begin{tabular}{|c|c|c|}
\hline \multicolumn{2}{|l|}{ Parameters } & Mean \pm SD \\
\hline \multicolumn{2}{|l|}{ Age (years) } & $30.38 \pm 9$ \\
\hline \multirow{5}{*}{$\begin{array}{l}\text { Haematologi- } \\
\text { cal parame- } \\
\text { ters }\end{array}$} & WBC $\times 10^{9} / \mathrm{L}$ & $10.42 \pm 6.58$ \\
\hline & $\mathrm{Hb}[\mathrm{g} / \mathrm{dL}]$ & $7.31 \pm 1.43$ \\
\hline & Platelets $\times 10^{9} / \mathrm{L}$ & $33.9 \pm 24.11$ \\
\hline & Reticulocyte count [\%] & $5.5 \% \pm 2.6 \%$ \\
\hline & Schistocytes cells [\%] & $6.14 \% \pm 2.26 \%$ \\
\hline \multirow{4}{*}{$\begin{array}{l}\text { Liver function } \\
\text { test }\end{array}$} & SGPT & $44.9 \pm 38.12$ \\
\hline & SGOT & $64.14 \pm 60.9$ \\
\hline & $\begin{array}{l}\text { Total bilirubin } \\
{[\mathrm{mg} / \mathrm{dL}]}\end{array}$ & $2.81 \pm 1.05$ \\
\hline & $\begin{array}{l}\text { Serum albumin } \\
{[\mathrm{mg} / \mathrm{dL}]}\end{array}$ & $3.62 \pm 0.71$ \\
\hline \multirow{3}{*}{$\begin{array}{l}\text { Renal fun- } \\
\text { ction test } \\
\text { LDH [U/L] }\end{array}$} & $\begin{array}{l}\text { Serum creatinine } \\
{[\mathrm{g} / \mathrm{dL}]}\end{array}$ & $3.21 \pm 3.12$ \\
\hline & & $1966.52 \pm 941.13$ \\
\hline & & No. [\%] \\
\hline \multirow{5}{*}{$\begin{array}{l}\text { Clinical pre- } \\
\text { sentations }\end{array}$} & Fever & $12(57.1 \%)$ \\
\hline & $\begin{array}{l}\text { Bleeding manifesta- } \\
\text { tion }\end{array}$ & $11(52.4 \%)$ \\
\hline & $\begin{array}{l}\text { Neurological manife- } \\
\text { station }\end{array}$ & $10(47.6 \%)$ \\
\hline & Renal impairment & 9 (42.9\%) \\
\hline & Cardiac manifestation & $2(9.5 \%)$ \\
\hline \multirow{2}{*}{$\begin{array}{l}\text { PLASMIC } \\
\text { score }\end{array}$} & 6 & $12(57.1 \%)$ \\
\hline & 7 & $9(42.9 \%)$ \\
\hline
\end{tabular}

SD - standard deviation; WBC - white blood cells; $\mathrm{Hb}$ - hemoglobin; SGPT - serum glutamic-pyruvic transaminase; SGOT - serum glutamic-oxaloacetic transaminase; LDH - lactate dehydrogenase

The patients were administered with steroids and TPEX for treatment, and the average number of sessions was 15 (range 2-45 sessions). Seven patients required rituximab, 6 of them received it at presentation, and it was administered as the second line in one patient; $47.62 \%$ of patients required cyclosporine as second or third line. There were 4 deaths (19.04\%), 2 of them were related to relapse and the other 2 were secondary to refractoriness to therapy.

The mean overall survival of the studied patients was 19.84 months [95\% (confidence interval) $\mathrm{Cl}$ : 16.41-23.55]. The patients presented with a PLASMIC score 6 and the absence of neurological problems were associated with better overall survival compared to other groups with significant $p=0.012,0.02$, respectively compared with other groups as shown figures 1 and 2 , while overall survival insignificantly correlated with age $>30$ years $(p=0.1)$, fever ( $p=0.42)$, and renal impairment $(p=0.38)$.

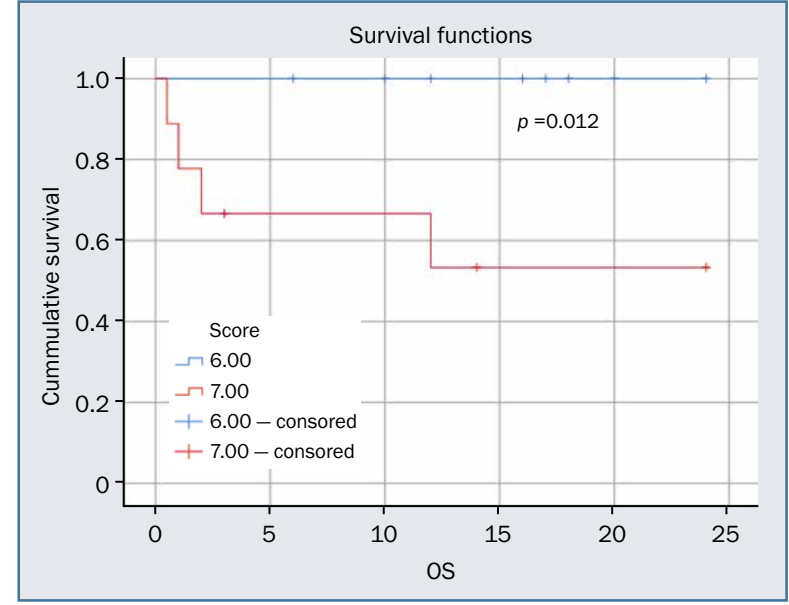

Figure 1. Effect of PLASMIC score on overall survival (OS) of studied cases

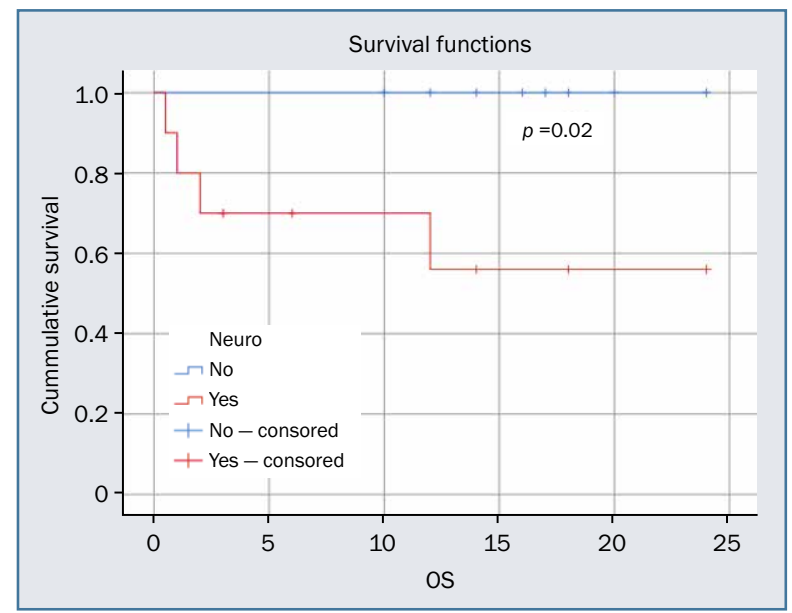

Figure 2. Effect of neurological (neuro) manifestations on overall survival (OS) of studied cases

\section{Discussion}

Thrombotic thrombocytopenic purpura's diagnosis is challenging secondary to its variable clinical presentations, which could overlap with other thrombotic microangiopathies and the unavailability of ADAMTS13 tests [12]. Many diagnostic scores are available such as the PLASMIC score, which is considered a useful application; as it has been able to diagnose TTP associated with ADAMTS-13 deficiency practically and statistically accurate, it's of low-cost, fast, especially in developing countries [10]. Other scores as the French registry-based score (Table III) and the one proposed by Bentley et al. are also available [13, 14].

In this study, we review the clinical presentations and laboratory tests for TTP. We also used the PLASMIC score to retrospectively assess our patients.

Our data revealed that females represented $81 \%$ of the studied cases, with a mean age of $30.45 \pm 9$ years. The most 
Table III. The French score - modified

\begin{tabular}{|l|c|}
\hline Variables & Points \\
\hline Platelet count $<3 \times 10^{10} / \mathrm{L}$ & 1 \\
Creatinine $>2.26 \mathrm{mg} / \mathrm{dL}$ & 1 \\
Reactive antinuclear antibodies & 1 \\
\hline
\end{tabular}

common feature in our study was fever (57.1\%), followed by bleeding manifestations ( $52.4 \%)$, neurological manifestations $(47.6 \%)$ in form of headache, slurred speech, seizures, confusion up to coma, renal impairment (42.9\%), and lastly cardiac manifestations (9.5\%).

Meanwhile, an Omanian study conducted on 38 TTP patients revealed that the patients' mean age was 36 years, $66 \%$ of patients were females. Their clinical presentation was: fever (63\%), renal impairment (50\%), bleeding (31\%), fits $(21 \%)$, high blood pressure reading $(21 \%)$, headache (15\%), confusion (15\%), postpartum presentation (15\%) and stroke (7.9\%) [15].

In comparison to the review from Oklahoma TTP-HUS Registry that studied 78 patients with acquired TTP, gastrointestinal symptoms were found recorded in $69 \%$, weakness in $63 \%$, bleeding or purpura in $54 \%$, major neurological findings including (coma, stroke, transient focal abnormalities, and seizures) were recorded in $41 \%$ of patients, while minor neurological findings including (confusion and headache) were found in $26 \%$ of patients and fever with chills in $10 \%[16,17]$.

Focusing on neurological and renal affection in TTP patients, their pathogenesis is mainly caused by the deposition of hyaline thrombi widely with fibroblastic infiltration and endothelial overlay found in the terminal arterioles and capillaries of multi-organs. Pre-occlusive pseudo-aneurysmal dilatation may be one of the mechanisms. These mechanisms explain the severity of these events in TTP patients and their consideration as independent risk factors for prognosis [18].

We retrospectively stratified our iTTP patients based on the PLASMIC score, and all of them were categorized as a high-risk group, 12 patients (57.1\%) were with a score of 6 and 9 patients (42.9\%) were with a score of score 7 . The PLASMIC score was developed and validated by a study conducted on 214 cases in the Harvard Thrombotic Microangiopathies Research Collaborative Registry [10]. In addition, the $100 \%$ diagnostic accuracy for PLASMIC score was confirmed by a study conducted in eight patients with iTTP, 4 cases with ADAMTS-13 activity $<10 \%$, had a PLASMIC score of 6 . The other 4 had ADAMTS- 13 activity $>10 \%$, had a $<6$ score. Also, the PLASMIC score predicted accurately their response to TPEx and the risk of long-term poor outcomes [19].

However, Prevel and his colleagues [5] provide evidence that the diagnosis of iTTP based on clinical scores aimed at predicting a severe ADAMTS-13 deficiency may be less accurate among older patients as organ damage in older patients following microthrombi formation may develop earlier than in younger patients, leading to earlier clinical manifestations with older age. In contrast, the organs of younger patients could tolerate microthrombi and ischemia, which leads to more aggressive cytopenias.

Steroids and TPEx were used for treatment, and the average number of sessions was 15; (range 2-45 sessions). Seven patients required rituximab, 6 of them received it at presentation, and it was administrated as the second line in one patient, $47.62 \%$ of patients required cyclosporine as the second or third line. There were 4 deaths $(19.04 \%), 2$ of them were related to relapse and the other 2 were secondary to refractoriness to therapy. However, we noticed that using rituximab as the first line in addition to TPEx and steroids improved the survival of our patients who had neurological manifestation at presentation.

Rituximab is a monoclonal lgG1 $k$ antibody, which is commonly used in treatment regimens for iTTP. Rituximab can prevent relapse and reduce mortality. Its half-life is about 2 to 3 weeks but ultimately depends on the underlying treated condition, presence of TPEx, and $\mathrm{CD} 2 \mathrm{O}^{+}$Iymphocyte load [20, 21]. A prospective study by Carden et al. 2020 [22] conducted on three patients studying the role of rituximab in iTPP, found that rituximab can eliminate all circulating $\mathrm{CD} 2 \mathrm{O}^{+} \mathrm{B}$ and $\mathrm{T}$ cells within 24 hours for each patient, despite uninterrupted daily TPEx through multiple mechanisms, including antibody-dependent cell-mediated cytotoxicity, complement-dependent cytotoxicity, and apoptosis.

Data revealed that the mean overall survival of our patients was 19.84 months (95\%Cl: 16.41-23.55). Patients presented with PLASMIC score 7 and absence of neurological manifestations were associated with better overall survival with significant $p=0.012$ and 0.02 , respectively, compared with the other groups, while the overall survival insignificantly correlated with age, fever and renal impairment ( $p=0.1,0.42$, and 0.38 , respectively). Similarly, it was reported in a study from the UK TTP Registry involving 292 patients that $24 \%$ of the patients who presented with a reduced Glasgow Coma Score were significantly associated with shorter overall survival compared to the other groups $(p<0.0001)$ [23].

A study conducted by Prevel et al. [5] on 411 patients showed that for patients aged more than 60 years, renal impairment; cardiac involvement, and total TPEx volume were independently associated with mortality. That is why physicians should be aware of atypical clinical presentations and for immediate intervention once suspected, which could improve the prognosis in iTTP patients.

\section{Conclusion}

The high cost of ADAMTS-13 testing makes it infeasible to do it for all patients in our country, and that is why we retrospectively applied the PLASMIC score trying to validate 
it in our community. A high PLASMIC score indicates immediate TPEx without losing time waiting for ADAMTS-13 level, while a low score, synchronized with low clinical probability, justifies further search for an alternate diagnosis in order to avoid overuse and associated complications of TPEx. We also aimed to focus the spot on neurological presentation of TTP patient correlating with worse survival and this may be put into consideration as a risk factor during deciding the treatment plan.

\section{Authors' contributions}

SE, EJ, RSS, YS - equally participated in collecting clinical, radiological, and statistical analysis. MIM, MAG - interpreted laboratory data. All authors - contributed to the study design, participated in writing and editing the final version of the manuscript, read and approved the final manuscript.

\section{Conflict of interest}

On behalf of all authors, the corresponding author states that there is no conflict of interest.

\section{Financial support}

This research did not receive any specific grant from funding agencies in the public, commercial, or not-for-profit sectors.

\section{Ethics}

The work described in this article has been carried out in accordance with The Code of Ethics of the World Medical Association (Declaration of Helsinki) for experiments involving humans; EU Directive 2010/63/EU for animal experiments; Uniform requirements for manuscripts submitted to biomedical journals.

\section{References}

1. Staley EM, Cao W, Pham HP, et al. Clinical factors and biomarkers predict outcome in patients with immune-mediated thrombotic thrombocytopenic purpura. Haematologica. 2019; 104(1): 166-175, doi: 10.3324/haematol.2018.198275, indexed in Pubmed: 30171022.

2. Gómez-De León A, Villela-Martínez LM, Yáñez-Reyes JM, et al. Advances in the treatment of thrombotic thrombocytopenic purpura: repurposed drugs and novel agents. Expert Rev Hematol. 2020; 13(5): 461-470, doi: 10.1080/17474086.2020.1750361, indexed in Pubmed: 32243196.

3. Coppo P, Cuker A, George JN. Thrombotic thrombocytopenic purpura: toward targeted therapy and precision medicine. Res Pract Thromb Haemost. 2019; 3(1): 26-37, doi: 10.1002/rth2.12160, indexed in Pubmed: 30656273.

4. Page EE, Kremer Hovinga JA, Terrell DR, et al. Thrombotic thrombocytopenic purpura: diagnostic criteria, clinical features, and long-term outcomes from 1995 through 2015. Blood Adv. 2017; 1(10): 590-600, doi: 10.1182/bloodadvances.2017005124, indexed in Pubmed: 29296701.

5. Prevel R, Roubaud-Baudron C, Gourlain S, et al. Prognostic and long-term survival of immune thrombotic thrombocytopenic purpura in older patients. Blood. 2019, doi: 10.1182/blood.2019000748.
6. Rock GA, Shumak KH, Buskard NA, et al. Comparison of plasma exchange with plasma infusion in the treatment of thrombotic thrombocytopenic purpura. Canadian Apheresis Study Group. N Engl J Med. 1991; 325(6): 393-397, doi: 10.1056/NEJM199108083250604, indexed in Pubmed: 2062330.

7. Zheng XL, Vesely SK, Cataland SR, et al. ISTH guidelines for treatment of thrombotic thrombocytopenic purpura. J Thromb Haemost. 2020; 18(10): 2496-2502, doi: 10.1111/jth.15010, indexed in Pubmed: 32914526.

8. Hanlon A, Metjian A. Caplacizumab in adult patients with acquired thrombotic thrombocytopenic purpura. Ther Adv Hematol. 2020; 11: 2040620720902904, doi: 10.1177/2040620720902904, indexed in Pubmed: 32095224.

9. Scully M, Cataland SR, Peyvandi F, et al. HERCULES Investigators. Caplacizumab treatment for acquired thrombotic thrombocytopenic purpura. N Engl J Med. 2019; 380(4): 335-346, doi: 10.1056/NEJMoa1806311, indexed in Pubmed: 30625070.

10. Bendapudi PK, Hurwitz S, Fry A, et al. Derivation and external validation of the PLASMIC score for rapid assessment of adults with thrombotic microangiopathies: a cohort study. Lancet Haematol. 2017; 4(4): e157-e164, doi: 10.1016/\$2352-3026(17)30026-1, indexed in Pubmed: 28259520.

11. Benhamou Y, Boelle PY, Baudin B, et al. Reference Center for Thrombotic Microangiopathies. Cardiac troponin-I on diagnosis predicts early death and refractoriness in acquired thrombotic thrombocytopenic purpura. Experience of the French Thrombotic Microangiopathies Reference Center. J Thromb Haemost. 2015; 13(2): 293-302, doi: 10.1111/jth.12790, indexed in Pubmed: 25403270.

12. Chiasakul T, Cuker A. Clinical and laboratory diagnosis of TTP: an integrated approach. Hematology Am Soc Hematol Educ Program. 2018; 2018(1): 530-538, doi: 10.1182/asheducation-2018.1.530, indexed in Pubmed: 30504354.

13. Coppo P, Schwarzinger M, Buffet M, et al. French Reference Center for Thrombotic Microangiopathies. Predictive features of severe acquired ADAMTS13 deficiency in idiopathic thrombotic microangiopathies: the French TMA reference center experience. PLoS One. 2010; 5(4): e10208, doi: 10.1371/journal.pone.0010208, indexed in Pubmed: 20436664

14. Bentley MJ, Wilson AR, Rodgers GM. Performance of a clinical prediction score for thrombotic thrombocytopenic purpura in an independent cohort. Vox Sang. 2013; 105(4): 313-318, doi: 10.1111/ /vox.12050, indexed in Pubmed: 23662653.

15. Hashmi KA, Dowaiki SA. Thrombotic thrombocytopenic purpura in oman; manifestations and outcome. Retrospective study. Blood. 2018; 132(Suppl 1): 5004-5004, doi: 10.1182/blood-2018-99-115227.

16. George JN, Cuker A. Acquired TTP: clinical manifestations and diagnosis. UpToDate. 2018. http://www.uptodate.com (July 2, 2020).

17. Page EE, Kremer Hovinga JA, Terrell DR, et al. Thrombotic thrombocytopenic purpura: diagnostic criteria, clinical features, and long-term outcomes from 1995 through 2015. Blood Adv. 2017; 1(10): 590-600, doi: 10.1182/bloodadvances.2017005124, indexed in Pubmed: 29296701.

18. Tsai HM. Pathophysiology of thrombotic thrombocytopenic purpura. Int J Hematol. 2010; 91(1): 1-19, doi: 10.1007/s12185-009-0476-1, indexed in Pubmed: 20058209.

19. Oliveira DS, Lima TG, Benevides FL, et al. Plasmic score applicability for the diagnosis of thrombotic microangiopathy associated with ADAMTS13-acquired deficiency in a developing country. Hematol Transfus Cell Ther. 2019; 41(2): 119-124, doi: 10.1016/j. htct.2018.10.002, indexed in Pubmed: 31079658. 
20. McDonald V, Manns K, Mackie IJ, et al. Rituximab pharmacokinetics during the management of acute idiopathic thrombotic thrombocytopenic purpura. J Thromb Haemost. 2010; 8(6): 1201-1208, doi: 10.1111/j.1538-7836.2010.03818.x, indexed in Pubmed: 20175870.

21. Scully M, McDonald V, Cavenagh J, et al. A phase 2 study of the safety and efficacy of rituximab with plasma exchange in acute acquired thrombotic thrombocytopenic purpura. Blood. 2011; 118(7): 1746-1753, doi: 10.1182/blood-2011-03-341131, indexed in Pubmed: 21636861.
22. Carden MA, Gaddh M, Hoskote A, et al. Rituximab leads to early elimination of circulating $C D 20+T$ and $B$ lymphocytes in patients with iTTP despite ongoing TPEx. Blood Adv. 2020; 4(3): 477-481, doi: 10.1182/ /bloodadvances.2019001148.

23. Alwan F, Vendramin C, Vanhoorelbeke K, et al. Presenting ADAMTS13 antibody and antigen levels predict prognosis in immune-mediated thrombotic thrombocytopenic purpura. Blood. 2017; 130(4): 466-471, doi: 10.1182/blood-2016-12-758656, indexed in Pubmed: 28576877. 